\title{
COMPARAISON DE POPULATIONS DE CARPE COMMUNE (CYPRINUS CARPIO) À TAILLE COMMERCIALE : INTÉRÊT D'UNE APPROCHE GLOBALE.
}

\author{
V. PEREIRA (1), D. VALLOD (2), B. FAUCONNEAU (3) et Y. BOUVET (4)
}

(1) Institut Supérieur d'Agriculture Rhône-Alpes, 31 place Bellecour, 69288 Lyon cedex 02, France.

(2) Association pour le Développement de l'Aquaculture et de la Pêche en Rhône-Alpes, 3 bis quai chauveau, B.P. 220, 69336 Lyon cedex 09, France.

(3) Institut National de la Recherche Agronomique, Laboratoire de Physiologie des Poissons, Campus de Beaulieu, 35042 Rennes, France.

(4) Université Claude Bernard, Laboratoire d'Écologie des Eaux Douces et des Grands Fleuves, 43 boulevard du 11 novembre, 69622 Villeurbanne, France.

\section{RÉSUMÉ}

Six populations de carpe commune (Cyprinus carpio) ont été comparées sur la base de quatre critères : morphologie, génétique, rendement de transformation et aspect des filets. Les provenances géographiques des différents lots étudiés correspondent aux principales régions de production piscicole françaises : Dombes, Forez, Brenne, Allier, Lorraine et Poitou-Charentes.

Une discrimination géographique des populations est possible mais elle repose sur une approche globale intégrant l'ensemble des paramètres. Les différences semblent essentiellement liées aux conditions environnementales et permettent de distinguer les lots issus des régions où l'élevage est plus intensif et traditionnellement centré sur la carpe (Dombes, Forez, Lorraine) et les lots correspondant à un élevage plus extensif et davantage orienté vers le marché du repeuplement (Allier, Brenne, Poitou-Charentes).

Mots-clés : morphologie, génétique, rendement de transformation, conditions d'élevage, Cyprinus carpio.

\section{COMPARISON OF COMMON CARP (CYPRINUS CARPIO) POPULATIONS AT COMMERCIAL SIZE : INTEREST OF A GLOBAL STUDY.}

\begin{abstract}
Six common carp populations (Cyprinus carpio) were compared with four criteria : morphology, genetic, transformation yield and fillets aspect. Geographic origins of the different stocks studied correspond to the main areas of french production : Dombes, Forez, Brenne, Allier, Lorraine and Poitou-Charentes.
\end{abstract}


Geographic identification of stocks was possible if it is based on an global analysis integrating all the parameters measured. Differences seemed to be essentially related to environmental conditions and allow to differentiate stocks from regions where traditional and intensive breeding is practice, and based on the carp production (Dombes, Forez, Lorraine) and those with a predominance of breeding other species for restocking market (Allier, Brenne, Poitou-Charentes).

Key-words : morphology, genetic, yield of transformation, breeding conditions, Cyprinus carpio.

\section{INTRODUCTION}

La France produit environ 6000 tonnes de carpe commune (Cyprinus carpio) par an dont plus de la moitié est destinée au repeuplement (LE QUERE, 1997). Traditionnellement le reste se répartit entre une consommation intérieure peu développée (vente directe sur les zones de production et auprès des minorités ethniques ou religieuses) et un marché à l'export essentiellement orienté vers l'Allemagne. Dans les années 80 , devant la baisse régulière de ces débouchés traditionnels, les producteurs de poissons d'étang ont exploré de nouveaux marchés pour la carpe. Des enquêtes fondées sur la diversification et une restructuration du marché local ont ainsi montré que les produits transformés, tels que le filet de carpe désarêté et pelé, étaient mieux acceptés par le consommateur.

La politique d'optimisation de la filière piscicole (intensification, amélioration des performances et du rendement de transformation) a alors été particulièrement vive en région Rhône-Alpes où la carpe représente 50 à $60 \%$ de la production en étangs.

Le rendement de transformation est cependant faible pour permettre, à lui seul, un développement réel de la filière (prix de revient de la matière première élevée, marges allouées aux producteurs trop faibles). Une amélioration des rendements envisageable d'après les données de la littérature (FAUCONNEAU et al., 1995) devrait avoir une influence positive sur les facteurs limitants décrits et pourrait permettre l'ouverture vers de nouveaux marchés et générer un développement économiquement viable.

La variabilité des rendements de transformation observée sur les carpes élevées en France peut résulter de facteurs génétiques et environnementaux. Concernant les facteurs génétiques, les populations de carpes françaises ont été potentiellement caractérisées (Dombes, Forez) dans les années 30 mais ont fait l'objet de nombreux échanges avec les pays d'Europe centrale quelques années avant la guerre de 1914 (DE DROUIN DE BOUVILLE, 1928a et b, 1930 ; DE NEUFBOURG, 1930 ; ROULE, 1932 ; LEVRAT, 1965). En conséquence, la structure génétique de ces populations françaises n'est pas connue comme c'est le cas pour les carpes élevées dans les pays de l'Est (IRNAZAROW et BIALOWAS, 1994 ; IRNAZAROW, 1995) et en Israël.

Concernant les facteurs environnementaux, les différentes populations de carpes françaises sont élevées dans des conditions de production très variées depuis des conditions extensives dans des systèmes de production très orientés vers le marché de repeuplement jusqu'à des conditions intensives dans des systèmes de production comprenant des écloseries, des étangs d'alevinage et de grossissement pour des produits orientés vers des marchés de consommation. Le degré d'isolement génétique des populations élevées dans ces différents systèmes de production et les conséquences sur les rendements de transformation ne sont pas connus. 
C'est dans ce contexte que s'inscrit cette étude dont l'objectif est de comparer des populations de carpes communes pêchées entre novembre et décembre 1994 dans les principales régions de production françaises. Cette comparaison repose sur différents rendements de transformation mais également sur les caractéristiques génétiques et morphométriques des poissons ainsi que sur l'aspect de leurs filets.

\section{MATÉRIEL ET MÉTHODES}

\subsection{Protocole expérimental}

\subsubsection{Matériel biologique}

L'étude est effectuée sur 210 carpes de type "Miroir " ayant un poids compris entre 1,3 et $1,7 \mathrm{~kg}$. Ils proviennent de six régions d'élevage françaises : Dombes, Forez, Poitou-Charentes, Allier, Brenne et Lorraine. La population Dombes est représentée par deux lots de 30 poissons issus d'un élevage traditionnel reposant sur la productivité naturelle du milieu et d'un élevage optimisé où une complémentation alimentaire est fournie aux poissons. Les autres populations sont représentées par un échantillon de 30 poissons issus d'un même élevage. L'âge des poissons a pu être déterminé par des techniques de squelettochronologie (MEUNIER et PASCAL, 1981) à partir du premier rayon épineux des nageoires dorsales et anales. Ainsi, l'échantillon étudié comprend des carpes de 2 ans (Dombes et Forez), 3 ans (Dombes, Lorraine et Poitou-Charentes) et 4 ans (Allier, Brenne).

\subsubsection{Analyse morphométrique}

Les poissons sont abattus par une électrocution de deux minutes et numérotés. Ils sont ensuite disposés sur un fond clair et mat puis photographiés avec un appareil photo numérique Canon ION RC-260, sous l'éclairage d'une lampe halogène. Chaque poisson est photographié en vue de dessus et vue de côté. Les photographies sont ensuite visualisées sur un écran d'ordinateur et un ensemble de points remarquables et de points de contour sont saisis à l'aide d'une tablette à digitaliser. À partir des coordonnées de ces points, 40 grandeurs, comprenant des distances et des surfaces, sont automatiquement calculées par le logiciel «Emmanuelle » ou «ECP " (LEFRANC, 1992a et b).
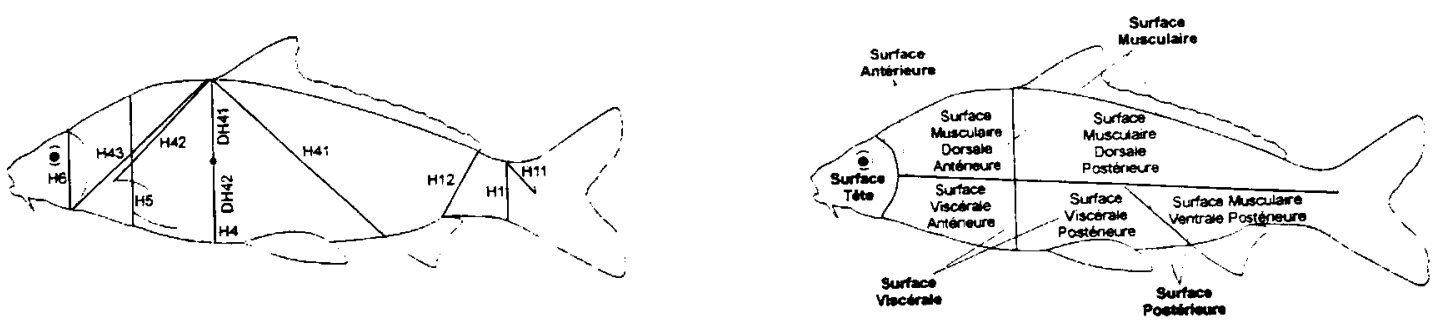

Figure 1

Hauteurs et surfaces utilisées pour l'analyse morphologique des différentes populations de carpes.

\section{Figure 1}

Heights and surfaces utilized for the morphological analysis of the different carp populations. 
Suivant la qualité des photos, tous les points remarquables n'ont pas pu être identifiés avec une précision suffisante. Ainsi, parmi les 40 mesures fournies par le logiciel, 31 ont été conservées et intégrées dans l'analyse morphologique (Figure 1 et Annexe 1).

\subsubsection{Filetage et parage}

Les carpes sont filetées au couteau par un même fileteur professionnel. La technique de filetage réalisée sur le poisson entier non éviscéré est décrite par HACKER dans COOPER (1987). Pour éviter l'influence de la raideur cadavérique sur le travail de la chair, le filetage est effectué dans les minutes qui suivent le sacrifice des poissons. Le parage, opération qui consiste à ôter la peau des filets, est mécanique.

\subsubsection{Pesée des organes}

Différents organes sont prélevés et pesés: les filets avec et sans peau, la tête, la carcasse étêtée, les viscères, les gonades et le foie.

\subsubsection{Description des filets}

Trois critères sont utilisés pour caractériser l'aspect des filets : leur hauteur, leur longueur et leur couleur. Les mesures de hauteur et de longueur sont réalisées à partir de photos numériques et du logiciel ECP. Le protocole de mesure est identique à celui suivi pour l'étude de la conformation externe des poissons. Les paramètres de couleur sont mesurés à l'aide d'un chromomètre (MINOLTA Cl 5000) en appliquant la tête de mesure de l'appareil sur le filet. Les mesures sont effectuées sur la partie dorsale et sur les deux faces du filet (3 mesures par face). La quantification des couleurs repose sur le système $L^{*} a^{*} b(C I E, 1976)$ qui comprend une estimation de la luminance $\left(L^{*}\right)$, traduisant la luminosité de la chair, et des composantes chromatiques de la couleur comprises respectivement entre le vert et le rouge $\left(a^{*}\right)$ et entre le bleu et le jaune $\left(b^{\star}\right)$.

\subsubsection{Analyse génétique}

L'étude génétique consiste en un examen de la variabilité de l'intensité relative des isoformes protéiques de différents systèmes enzymatiques par la technique de l'électrophorèse sur gel d'amidon.

In situ, les prélèvements de quatre tissus (muscle, foie, coeur et oeil) sont effectués sur chaque poisson et traités selon le protocole décrit par PASTEUR et al. (1987), adapté par BOUVET et al. (1991). Au total, 33 loci ont été révélés codant pour 17 systèmes

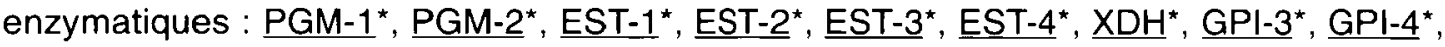

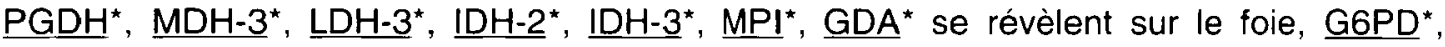

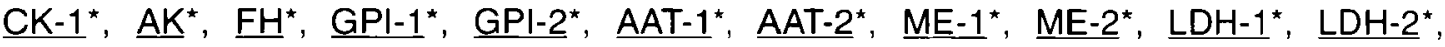

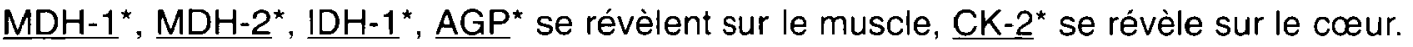

\subsection{Analyse des données}

\subsubsection{Transformation des données initiales}

Les données morphologiques sont standardisées afin d'éviter les variations de taille liées aux différences d'âge entre les populations. Cette standardisation repose sur la relation d'allométrie entre les données morphologiques et la taille du poisson (GOULD, 1966). Les coefficients d'allométrie sont calculés pour chacune des grandeurs et pour chaque population par une régression non linéaire sur le poids. Ils correspondent à la 
pente de la régression linéaire sur les données transformées en logarithme. Les mesures à un même poids moyen $\mathrm{Pm}(1,5 \mathrm{~kg})$ sont ensuite rétrocalculées pour chacun des poissons selon la formule :

$Y m=1 i(P m / P i)$

Ym : Grandeur moyenne correspondant au poids moyen ;

$\mathrm{Pi} \quad$ : Poids de l'individu ;

Yi : Grandeur de l'individu.

Les données issues de la pesée des organes sont intégrées dans le calcul d'indices somatiques et de rendements de transformation (Tableau I).

\section{Tableau I}

Indices somatiques et rendements de transformation calculés à partir des données pondérales. $[P V=$ poids vif $(\mathrm{g}), \mathrm{L}=$ longueur standard $(\mathrm{cm})]$.

\section{Table I}

Somatic index and transformation yields calculated from weight data. $[P V=$ total weight $(\mathbf{g}), \mathrm{L}=$ standard length $(\mathrm{cm})]$.

\begin{tabular}{|c|c|c|}
\hline Indices somatiques & $\begin{array}{l}\text { Coefficient de condition } \\
\text { Indice viscéro-somatique } \\
\text { Indice hépato-somatique } \\
\text { Indice gonado-somatique }\end{array}$ & $\begin{array}{l}(P V \times 100) / L^{3} \\
\left(P_{\text {visceres }} \times 100\right) / P V \\
\left(P_{\text {tore }} \times 100\right) / P V \\
\left(P_{\text {jonases }} \times 100\right) / P V\end{array}$ \\
\hline $\begin{array}{c}\text { Rendements } \\
\text { de transformation }\end{array}$ & $\begin{array}{l}\text { Rendement au filetage } \\
\text { Rendement à l'étêtage } \\
\text { Rendement en carcasse étêtée }\end{array}$ & 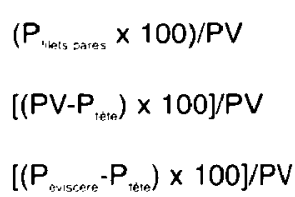 \\
\hline
\end{tabular}

\subsubsection{Traitements statistiques}

Pour les données génétiques, ont été considérées successivement :

- la variabilité intra-population évaluée par le taux de polymorphisme et d'hétérozygotie ainsi qu'ils sont définis par PASTEUR (1985) ;

- la distance génétique entre populations illustrées par une Analyse des Correspondances Multiples (ACM) appliquée à l'ensemble des génotypes individuels.

Pour les critères morphologiques, somatiques et les paramètres décrivant l'aspect des filets, le traitement des données comprend le calcul d'éléments statistiques élémentaires (moyenne et erreur standard), des analyses de variance, des Analyses en Composantes Principales (ACP) et des Analyses Factorielles Discriminantes (AFD). Les analyses de variance, appliquées à chacune des variables, permettent de tester les 
différences entre lots, provenances géographiques et sexes. Les ACP sont utilisées pour quantifier les liaisons entre variables, visualiser la variabilité intra- et inter-lots puis faire une sélection des variables à intégrer dans les analyses discriminantes. Ces dernières, en optimisant les variabilités inter-lots, permettent d'illustrer les différences et ressemblances entre populations et de définir les variables les plus discriminantes.

\section{RÉSULTATS}

\subsection{Caractéristiques génétiques}

Sur les 33 loci étudiés, 5 seulement se sont révélés polymorphes: $\mathrm{PGM}-1^{*}$, $\mathrm{MDH} 3^{*}, \underline{\mathrm{CK}-1^{*}}, \mathrm{LDH} 3^{*}$ et $\mathrm{MPI}^{*}$. Chaque système enzymatique est représenté, pour chacune des souches, par le même nombre de loci et par le même allèle commun. La relation de dominance des allèles n'est donc pas un bon critère pour comparer les lots. Néanmoins, quelques mutations apparaissent et permettent de caractériser les souches par leur richesse génotypique. Les taux d'hétérozygotie sont faibles mais présentent des différences selon les souches (Figure 2). Ainsi, la souche du Forez est la moins différenciée et son pool génique se retrouve dans toutes les autres souches. En revanche, la population Poitou-Charentes est caractérisée par une plus grande variabilité génétique. Toutefois, les fréquences de ces mutations restent trop faibles pour permettre d'obtenir des axes factoriels discriminant fortement les souches par leur composition allèlique (Figure 3).

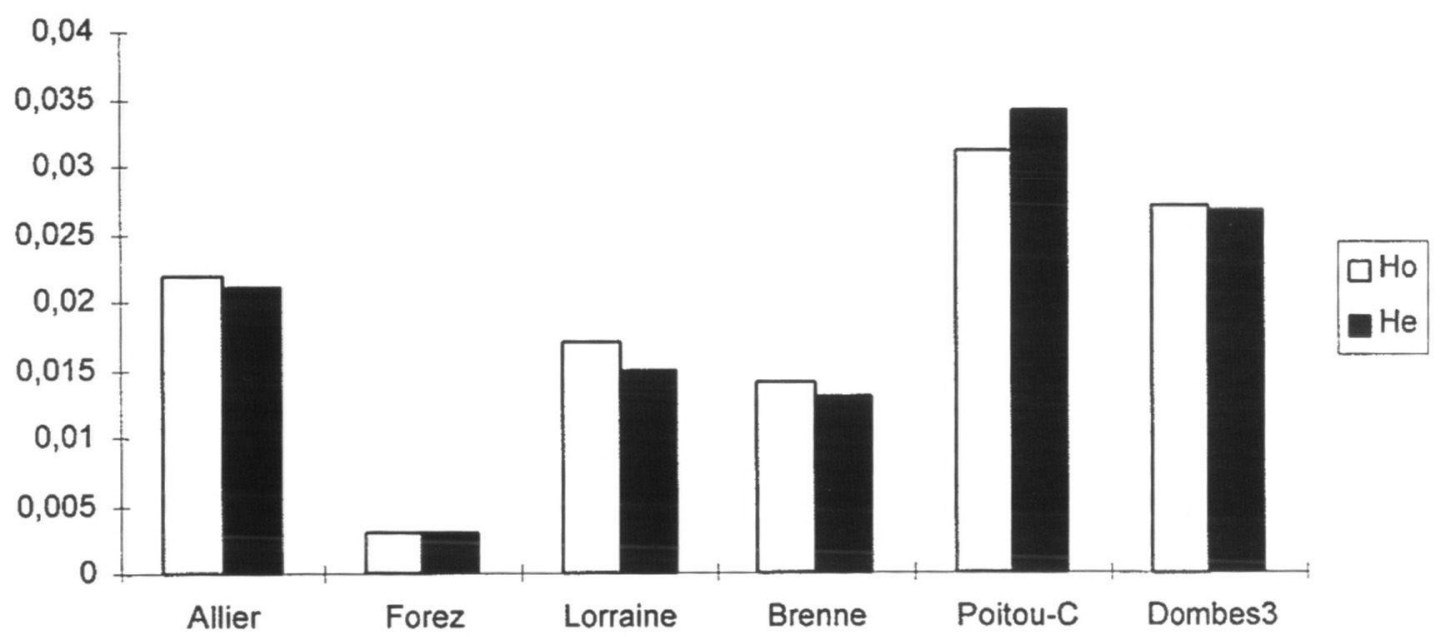

Figure 2

Variation des taux d'hétérozygotie observés $(\mathrm{Ho})$ et théoriques $(\mathrm{He})$ dans les différentes zones de production. Les taux d'hétérozygotie théoriques sont calculés à partir de la loi de HARDY-WEINBERG.

\section{Figure 2}

Variation of observed $(\mathrm{Ho})$ and theoric $(\mathrm{He})$ heterozygoty rates in the different aeras of production. Heterozygoty rates are calculated from HARDY-WEINBERG law. 


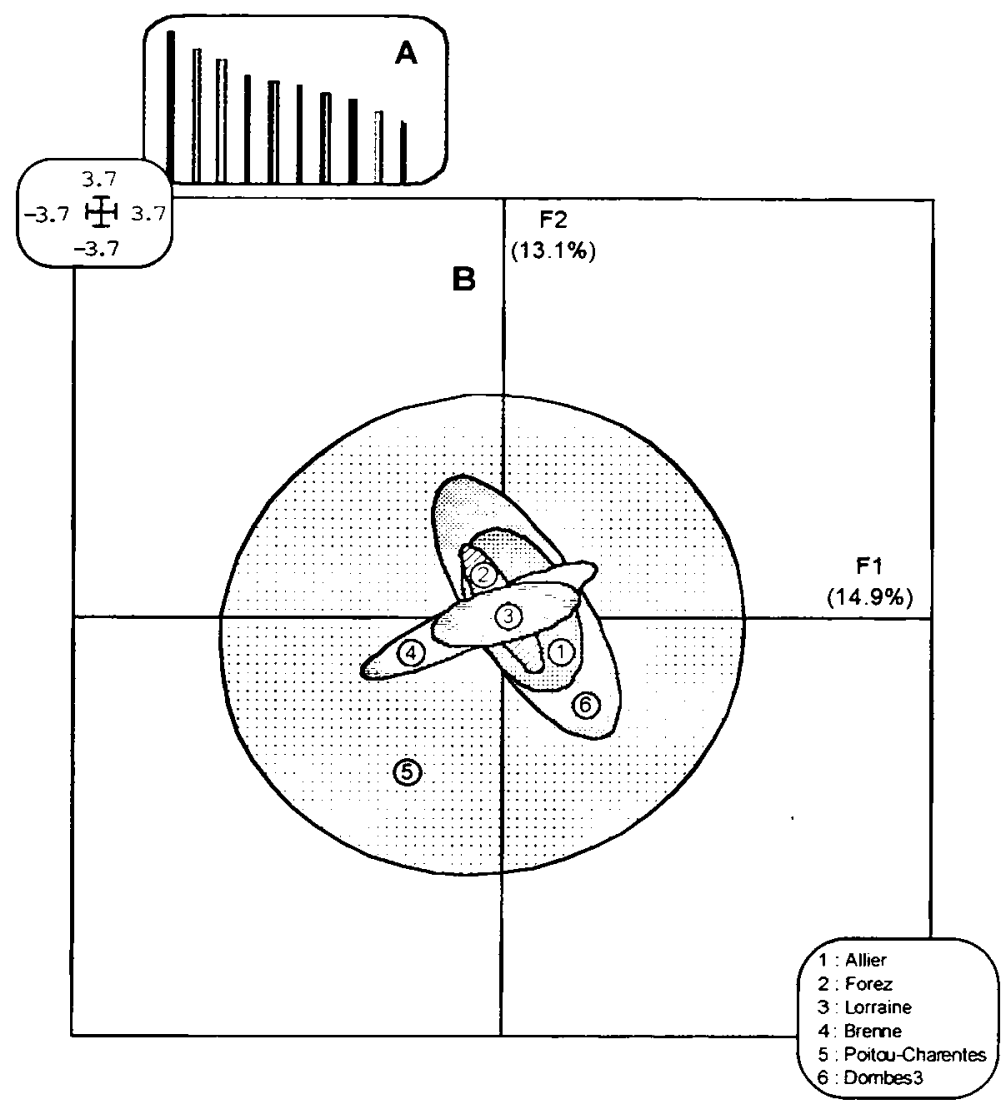

Figure 3

Résultats de l'Analyse des Correspondances Multiples réalisée sur le tableau des caractéristiques génétiques.

(A) Histogramme des valeurs propres.

(B) Distribution des lots dans le plan factoriel F1-F2, chaque lot est positionné au centre de gravité de ses individus.

\section{Figure 3}

Results of the Multiple Correspondence Analysis performed on the genetic characteristics data set.

(A) Histogram of eigenvalues.

(B) Distribution of stocks on the F1-F2 factorial plane, each stock is located at the weighted average of specimens.

\subsection{Caractéristiques morphologiques}

L'analyse discriminante est réalisée sur 17 variables, sélectionnées à partir des analyses de variance et ACP (PEREIRA, 1996). Le tableau des valeurs moyennes de ces variables permet d'apprécier la finesse des différences morphologiques entre les lots (Tableau II). Néanmoins, il existe des tendances qui associent des différences dans le développement relatif de la partie antérieure, notamment de la tête, et dans le degré de compacité du poisson (Figure 4). Ainsi, les poissons de l'Allier, de la Dombes (2 ans) et de la Lorraine se distinguent des autres lots par une croissance limitée de la tête et un développement en hauteur de la partie antérieure plus important. De même, les poissons de l'Allier, de la Brenne et de Poitou-Charentes se caractérisent par une silhouette plus allongée. 
Les critères morphologiques étudiés fournissent donc une grille de discrimination des populations satisfaisante même s'ils ne sont pas suffisants pour aboutir à une discrimination stricte.

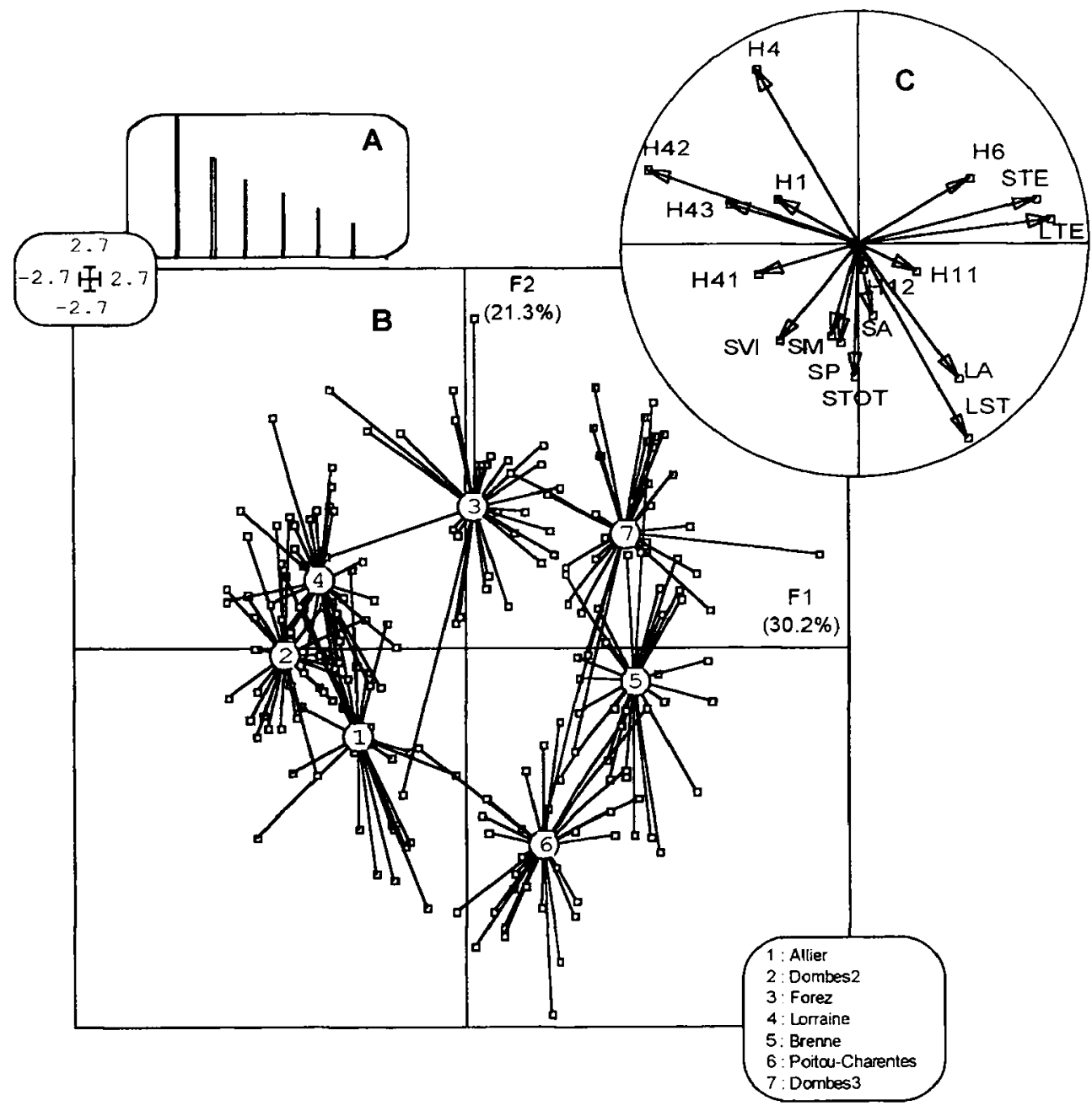

Figure 4

Résultats de l'Analyse factorielle Discriminante réalisée sur le tableau des données morphologiques standardisées.

(A) Histogramme des valeurs propres.

(B) Distribution des lots dans le plan factoriel F1-F2, chaque lot est positionné au centre de gravité de ses individus.

(C) Coefficients de corrélation des variables canoniques avec les variables initiales.

Figure 4

Results of the factorial Discriminant Analysis performed on the morphological data set.

(A) Histogram of eigenvalues.

(B) Distribution of stocks on the F1-F2 factorial plane, each stock is located at the weighted average of specimens.

(C) Correlation coefficients of canonical variables with initial variables. 
Tableau II

Moyennes ( $\pm 2 \times$ erreur standard) des variables morphologiques mesurées sur les carpes issues des différentes zones de production et de poids homogène de $1,5 \mathrm{~kg}$. Les surfaces sont exprimées en $\mathrm{cm}^{2}$, les longueurs et hauteurs en $\mathrm{cm}$.

Table II

Means ( $\pm 2 \times$ erreur standard error) of morphological variables measured on carps come from different areas of production and with an homogeneous weight of $1.5 \mathrm{~kg}$. Surfaces are express in $\mathrm{cm}^{2}$, lengths and heights in $\mathrm{cm}$.

\begin{tabular}{|c|c|c|c|c|c|c|c|}
\hline & Allier & Dombes2 & Forez & Lorraine & Brenne & Poitou-C & Dombes3 \\
\hline $\begin{array}{c}\text { Surface } \\
\text { totale }\end{array}$ & $\begin{array}{r}380,4 \\
\pm 9,5\end{array}$ & $\begin{array}{l}372,3 \\
\pm 4,6\end{array}$ & $\begin{array}{l}358,8 \\
\pm 3,4\end{array}$ & $\begin{array}{l}362,6 \\
\pm 2,5\end{array}$ & $\begin{array}{l}367,9 \\
\pm 7,1\end{array}$ & $\begin{array}{l}380,3 \\
\pm 3,7\end{array}$ & $\begin{array}{c}369,7 \\
\pm 4\end{array}$ \\
\hline $\begin{array}{l}\text { Surface } \\
\text { tête }\end{array}$ & $\begin{array}{r}44,67 \\
\pm 1,9\end{array}$ & $\begin{array}{r}42,16 \\
\pm 1,2\end{array}$ & $\begin{array}{l}47,91 \\
\pm 1,4\end{array}$ & $\begin{array}{r}45,6 \\
\pm 1,3\end{array}$ & $\begin{array}{r}52,8 \\
\pm 1,5\end{array}$ & $\begin{array}{r}49,1 \\
\pm 1,6\end{array}$ & $\begin{array}{c}56 \\
\pm 1,5\end{array}$ \\
\hline $\begin{array}{l}\text { Surface } \\
\text { viscérale }\end{array}$ & $\begin{array}{l}125,4 \\
\pm 3,9\end{array}$ & $\begin{array}{l}125,4 \\
\pm 1,6\end{array}$ & $\begin{array}{l}118,8 \\
\pm 2,1\end{array}$ & $\begin{array}{l}120,3 \\
\pm 1,8\end{array}$ & $\begin{array}{l}121 \\
\pm 2\end{array}$ & $\begin{array}{l}122,4 \\
\pm 1,9\end{array}$ & $\begin{array}{r}116,5 \\
\pm 1,8\end{array}$ \\
\hline $\begin{array}{l}\text { Surface } \\
\text { musculaire }\end{array}$ & $\begin{array}{l}210,1 \\
\pm 5,4\end{array}$ & $\begin{array}{l}204,8 \\
\pm 3,8\end{array}$ & $\begin{array}{l}192,1 \\
\pm 2,9\end{array}$ & $\begin{array}{l}196,7 \\
\pm 2,4\end{array}$ & $\begin{array}{l}194,2 \\
\pm 6,1\end{array}$ & $\begin{array}{l}208,8 \\
\pm 2,6\end{array}$ & $\begin{array}{r}197,2 \\
\pm 3,5\end{array}$ \\
\hline $\begin{array}{c}\text { Surface } \\
\text { antérieure }\end{array}$ & $\begin{array}{c}158,9 \\
\pm 5\end{array}$ & $\begin{array}{l}163,4 \\
\pm 2,9\end{array}$ & $\begin{array}{l}159,3 \\
\pm 3,3\end{array}$ & $\begin{array}{r}155 \\
\pm 3,4\end{array}$ & $\begin{array}{l}158,5 \\
\pm 3,6\end{array}$ & $\begin{array}{l}167,1 \\
\pm 2,4\end{array}$ & $\begin{array}{l}160,3 \\
\pm 2,8\end{array}$ \\
\hline $\begin{array}{c}\text { Surtace } \\
\text { postérieure }\end{array}$ & $\begin{array}{r}221,4 \\
\pm 6,3\end{array}$ & $\begin{array}{l}209,5 \\
\pm 3,3\end{array}$ & $\begin{array}{l}199,6 \\
\pm 3,7\end{array}$ & $\begin{array}{l}207,6 \\
\pm 3,6\end{array}$ & $\begin{array}{l}209,3 \\
\pm 6,4\end{array}$ & $\begin{array}{l}213,1 \\
\pm 2,8\end{array}$ & $\begin{array}{c}209,5 \\
\pm 4\end{array}$ \\
\hline $\begin{array}{l}\text { Longueur } \\
\text { tête }\end{array}$ & $\begin{array}{c}9,4 \\
\pm 0,2\end{array}$ & $\begin{array}{c}8,8 \\
\pm 0,2\end{array}$ & $\begin{array}{c}9,9 \\
\pm 0,1\end{array}$ & $\begin{array}{c}9,3 \\
\pm 0,2\end{array}$ & $\begin{array}{r}10,6 \\
\pm 0,2\end{array}$ & $\begin{array}{r}10,1 \\
\pm 0,2\end{array}$ & $\begin{array}{r}10,7 \\
\pm 0,2\end{array}$ \\
\hline $\begin{array}{l}\text { Longueur } \\
\text { antérieure }\end{array}$ & $\begin{array}{r}16,4 \\
\pm 0,4\end{array}$ & $\begin{array}{r}16,7 \\
\pm 0,2\end{array}$ & $\begin{array}{r}16,4 \\
\pm 0,3\end{array}$ & $\begin{array}{r}16,2 \\
\pm 0,2\end{array}$ & $\begin{array}{r}17,2 \\
\pm 0,2\end{array}$ & $\begin{array}{r}17,9 \\
\pm 0,2\end{array}$ & $\begin{array}{r}16,9 \\
\pm 0,2\end{array}$ \\
\hline $\begin{array}{l}\text { Longueur } \\
\text { standard }\end{array}$ & $\begin{array}{r}36,6 \\
\pm 0,7\end{array}$ & $\begin{array}{r}34,9 \\
\pm 0,4\end{array}$ & $\begin{array}{l}34,2 \\
\pm 0,5\end{array}$ & $\begin{array}{l}34,0 \\
\pm 0,4\end{array}$ & $\begin{array}{r}37,3 \\
\pm 0,5\end{array}$ & $\begin{array}{r}38,7 \\
\pm 0,4\end{array}$ & $\begin{array}{r}35,8 \\
\pm 0,4\end{array}$ \\
\hline Hauteur 1 & $\begin{array}{c}6,1 \\
\pm 0,2\end{array}$ & $\begin{array}{c}6,1 \\
\pm 0,1\end{array}$ & $\begin{array}{c}5,7 \\
\pm 0,1\end{array}$ & $\begin{array}{c}6 \\
\pm 0,1\end{array}$ & $\begin{array}{l}6 \\
\pm 0,1\end{array}$ & $\begin{array}{c}5,5 \\
\pm 0,1\end{array}$ & $\begin{array}{c}5,8 \\
\pm 0,1\end{array}$ \\
\hline Hauteur 11 & $\begin{array}{c}4 \\
\pm 0,2\end{array}$ & $\begin{array}{c}3,5 \\
\pm 0,2\end{array}$ & $\begin{array}{c}3,7 \\
\pm 0,2\end{array}$ & $\begin{array}{c}3,4 \\
\pm 0,1\end{array}$ & $\begin{array}{c}3,7 \\
\pm 0,2\end{array}$ & $\begin{array}{c}3,7 \\
\pm 0.2\end{array}$ & $\begin{array}{c}3,8 \\
\pm 0,2\end{array}$ \\
\hline Hauteur 12 & $\begin{array}{c}6,2 \\
\pm 0,1\end{array}$ & $\begin{array}{c}6,1 \\
\pm 0,1\end{array}$ & $\begin{array}{c}6,2 \\
\pm 0,1\end{array}$ & $\begin{array}{c}6,1 \\
\pm 0,1\end{array}$ & $\begin{array}{c}6,2 \\
\pm 0,1\end{array}$ & $\begin{array}{c}6,2 \\
\pm 0,2\end{array}$ & $\begin{array}{c}6,1 \\
\pm 0,1\end{array}$ \\
\hline Hauteur 4 & $\begin{array}{c}16 \\
\pm 0,3\end{array}$ & $\begin{array}{l}16,2 \\
\pm 0,1\end{array}$ & $\begin{array}{r}16,4 \\
\pm 0,2\end{array}$ & $\begin{array}{r}16,3 \\
\pm 0,2\end{array}$ & $\begin{array}{r}15,3 \\
\pm 0,2\end{array}$ & $\begin{array}{r}14,5 \\
\pm 0,2\end{array}$ & $\begin{array}{c}16 \\
\pm 0,2\end{array}$ \\
\hline Hauteur 41 & $\begin{array}{r}18,5 \\
\pm 0,3\end{array}$ & $\begin{array}{r}18,3 \\
\pm 0,2\end{array}$ & $\begin{array}{r}17,7 \\
\pm 0,2\end{array}$ & $\begin{array}{r}18,2 \\
\pm 0,2\end{array}$ & $\begin{array}{r}17,7 \\
\pm 0,2\end{array}$ & $\begin{array}{r}17,8 \\
\pm 0,2\end{array}$ & $\begin{array}{r}17,9 \\
\pm 0,2\end{array}$ \\
\hline Hauteur 42 & $\begin{array}{r}15,9 \\
\pm 0,4\end{array}$ & $\begin{array}{r}16,2 \\
\pm 0,1\end{array}$ & $\begin{array}{r}15,2 \\
\pm 0,3\end{array}$ & $\begin{array}{r}16,3 \\
\pm 0,2\end{array}$ & $\begin{array}{l}13,7 \\
\pm 0.2\end{array}$ & $\begin{array}{r}13,7 \\
\pm 0,2\end{array}$ & $\begin{array}{r}14,2 \\
\pm 0,2\end{array}$ \\
\hline Hauteur 43 & $\begin{array}{r}16,7 \\
\pm 0,3\end{array}$ & $\begin{array}{r}16,9 \\
\pm 0,2\end{array}$ & $\begin{array}{r}16,5 \\
\pm 0,2\end{array}$ & $\begin{array}{r}16,9 \\
\pm 0,2\end{array}$ & $\begin{array}{c}16 \\
\pm 0,2\end{array}$ & $\begin{array}{r}16,2 \\
\pm 0,2\end{array}$ & $\begin{array}{r}16,4 \\
\pm 0,2\end{array}$ \\
\hline Hauteur 6 & $\begin{array}{c}9,4 \\
\pm 0,2\end{array}$ & $\begin{array}{c}9,4 \\
\pm 0,2\end{array}$ & $\begin{array}{c}9,7 \\
\pm 0,2\end{array}$ & $\begin{array}{c}9,7 \\
\pm 0,2\end{array}$ & $\begin{array}{c}9,9 \\
\pm 0,1\end{array}$ & $\begin{array}{r}9,7 \\
\pm 0,1\end{array}$ & $\begin{array}{r}10,3 \\
\pm 0,1\end{array}$ \\
\hline
\end{tabular}




\subsection{Caractéristiques des filets}

Les critères de couleur et de conformation des filets varient selon les lots mais ils ne permettent pas de discriminer nettement les individus des différentes populations (Tableau III). Ce sont les filets des poissons issus de la Dombes et du Forez qui ont les caractéristiques les plus proches (Figure 5). Les caractéristiques des filets des poissons de Lorraine sont assez proches de celle des poissons de ce premier groupe.

Les différences entre les poissons des autres provenances géographiques concernent essentiellement la hauteur des filets, la luminance sur la face externe et l'indice de teinte rouge sur les faces externes et internes. Les filets des poissons de Poitou-Charentes sont caractérisés par une hauteur faible et une coloration rouge importante par rapport aux filets des poissons de la Lorraine. Les filets des poissons de l'Allier et de la Brenne se distinguent des autres par leur faible luminance sur la face externe.

\section{Tableau III}

Moyennes ( $\pm 2 \times$ erreur standard) des données décrivant l'aspect des filets. Les longueurs et hauteurs de filet sont exprimées en $\mathrm{mm}$ et sont relatives à des carpes de poids homogène de $1,5 \mathrm{~kg}$. Les données de coloration sont sans unité.

\section{Table III}

Means ( $\pm 2 \times$ standard error) of data describing fillets aspect. Fillet lengths and heights are express in $\mathrm{mm}$ and are relative to carps with an homogeneous weight of $1.5 \mathrm{~kg}$. Data of coloring are without unity.

\begin{tabular}{|c|c|c|c|c|c|c|c|}
\cline { 2 - 7 } \multicolumn{1}{c|}{} & Allier & Dombes2 & Forez & Lorraine & Brenne & Poitou-C & Dombes3 \\
\hline $\begin{array}{c}\text { Longueur } \\
\text { filet }\end{array}$ & $\begin{array}{c}260 \\
\pm 7\end{array}$ & $\begin{array}{c}246 \\
\pm 4\end{array}$ & $\begin{array}{c}224 \\
\pm 5\end{array}$ & $\begin{array}{c}221 \\
\pm 4\end{array}$ & $\begin{array}{c}248 \\
\pm 6\end{array}$ & $\begin{array}{c}268 \\
\pm 6\end{array}$ & $\begin{array}{c}226 \\
\pm 6\end{array}$ \\
\hline $\begin{array}{c}\text { Hauteur } \\
\text { filet }\end{array}$ & $\begin{array}{c}130 \\
\pm 3\end{array}$ & $\begin{array}{c}133 \\
\pm 3\end{array}$ & $\begin{array}{c}134 \\
\pm 4\end{array}$ & $\begin{array}{c}139 \\
\pm 3\end{array}$ & $\begin{array}{c}130 \\
\pm 3\end{array}$ & $\begin{array}{c}121 \\
\pm 3\end{array}$ & $\begin{array}{c}133 \\
\pm 3\end{array}$ \\
\hline $\begin{array}{c}\text { Luminance } \\
\text { face externe }\end{array}$ & $\begin{array}{c}41,40 \\
\pm 0,51\end{array}$ & $\begin{array}{c}46,41 \\
\pm 0,78\end{array}$ & $\begin{array}{c}46,41 \\
\pm 1,14\end{array}$ & $\begin{array}{c}40,47 \\
\pm 0,74\end{array}$ & $\begin{array}{c}47,90 \\
\pm 0,88\end{array}$ & $\begin{array}{c}46,93 \\
\pm 1,06\end{array}$ & $\begin{array}{c}45,69 \\
\pm 1,02\end{array}$ \\
\hline $\begin{array}{c}\text { Luminance } \\
\text { face interne }\end{array}$ & $\begin{array}{c}39,16 \\
\pm 0,59\end{array}$ & $\begin{array}{c}42,46 \\
\pm 0,51\end{array}$ & $\begin{array}{c}40,57 \\
\pm 0,44\end{array}$ & $\begin{array}{c}42,04 \\
\pm 0,69\end{array}$ & $\begin{array}{c}41 \\
\pm 0,50\end{array}$ & $\begin{array}{c}41,32 \\
\pm 0,57\end{array}$ & $\begin{array}{c}42,58 \\
\pm 0,46\end{array}$ \\
\hline $\begin{array}{c}\text { Coloration rouge } \\
\text { face externe }\end{array}$ & $\begin{array}{c}10,48 \\
\pm 0,60\end{array}$ & $\begin{array}{c}9,17 \\
\pm 0,58\end{array}$ & $\begin{array}{c}9,58 \\
\pm 0,90\end{array}$ & $\begin{array}{c}13,27 \\
\pm 0,54\end{array}$ & $\begin{array}{c}6,82 \\
\pm 0,48\end{array}$ & $\begin{array}{c}11,60 \\
\pm 1,02\end{array}$ & $\begin{array}{c}11,38 \\
\pm 0,74\end{array}$ \\
\hline $\begin{array}{c}\text { Coloration rouge } \\
\text { face interne }\end{array}$ & $\begin{array}{c}5,60 \\
\pm 0,43\end{array}$ & $\begin{array}{c}4,12 \\
\pm 0,38\end{array}$ & $\begin{array}{c}5,05 \\
\pm 0,34\end{array}$ & $\begin{array}{c}4,78 \\
\pm 0,44\end{array}$ & $\begin{array}{c}2,94 \\
\pm 0,45\end{array}$ & $\begin{array}{c}5,39 \\
\pm 0,49\end{array}$ & $\begin{array}{c}4,36 \\
\pm 0,40\end{array}$ \\
\hline $\begin{array}{c}\text { Coloration jaune } \\
\text { face externe }\end{array}$ & $\begin{array}{c}3,07 \\
\pm 0,39\end{array}$ & $\begin{array}{c}4,06 \\
\pm 0,31\end{array}$ & $\begin{array}{c}2,87 \\
\pm 0,46\end{array}$ & $\begin{array}{c}2,55 \\
\pm 0,29\end{array}$ & $\begin{array}{c}1,89 \\
\pm 0,43\end{array}$ & $\begin{array}{c}3,27 \\
\pm 0,41\end{array}$ & $\begin{array}{c}4,36 \\
\pm 0,41\end{array}$ \\
\hline $\begin{array}{c}\text { Coloration jaune } \\
\text { face interne }\end{array}$ & $\begin{array}{c}2,84 \\
\pm 0,25\end{array}$ & $\begin{array}{c}3,09 \\
\pm 0,19\end{array}$ & $\begin{array}{c}2,54 \\
\pm 0,22\end{array}$ & $\begin{array}{c}2,71 \\
\pm 0,25\end{array}$ & $\begin{array}{c}2,20 \\
\pm 0,23\end{array}$ & $\begin{array}{c}1,79 \\
\pm 0,25\end{array}$ & $\begin{array}{c}2,77 \\
\pm 0,29\end{array}$ \\
\hline
\end{tabular}




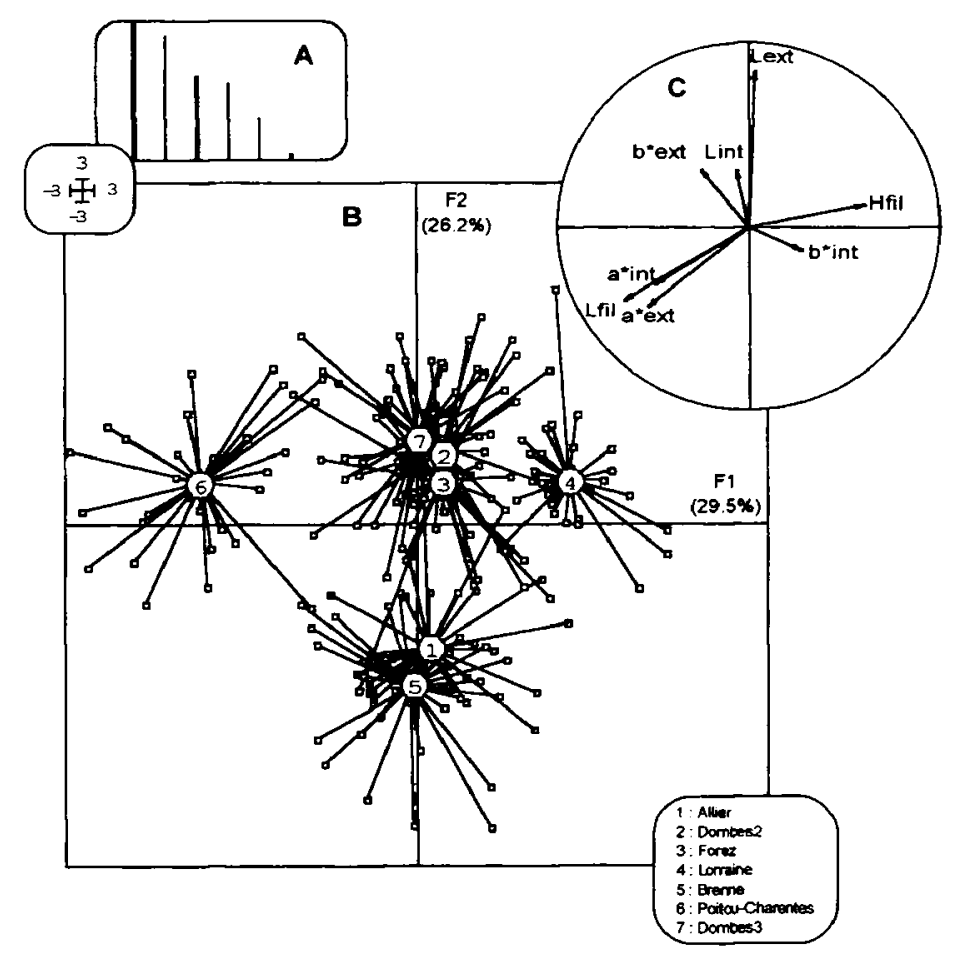

Figure 5

Résultats de l'Analyse factorielle Discriminante réalisée sur le tableau des données liées à l'aspect des filets.

(A) Histogramme des valeurs propres.

(B) Distribution des lots dans le plan factoriel F1-F2, chaque lot est positionné au centre de gravité de ses individus.

(C) Coefficients de corrélation des variables canoniques avec les variables initiales.

\section{Figure 5}

Results of the factorial Discriminant Analysis performed on the fillet aspects data set.

(A) Histogram of eigenvalues.

(B) Distribution of stocks on the F1-F2 factorial plane, each stock is located at the weighted average of specimens.

(C) Correlation coefficients of canonical variables with initial variables.

\subsection{Indices somatiques et rendements de transformation}

Les paramètres considérés varient selon l'origine géographique des poissons mais ils sont caractérisés par une forte variabilité individuelle (Tableau IV). Aucun lot ne peut donc être différencié nettement des autres sur la base des indices physiologiques ou des rendements de transformation. II existe néanmoins des différences impliquant trois variables : l'indice hépato-somatique, le coefficient de condition et, dans une moindre mesure, le rendement au filetage (Figure 6). Ainsi, les poissons de la Dombes (2 ans), de la Lorraine et du Forez, ayant un coefficient de condition et un indice hépato-somatique élevés, s'opposent aux poissons de l'Allier, de la Brenne et de Poitou-Charentes. Les rendements de filetage des poissons de Poitou-Charentes sont plus élevés que ceux des poissons de la Brenne et de la Dombes ( 3 ans). 


\section{Tableau IV}

Moyennes ( $\pm 2 \times$ erreur standard) des indices somatiques et rendements de transformation obtenus dans les différentes zones de production. Les indices et rendements sont exprimés en pourcentage.

\section{Table IV}

Means ( $\pm 2 \times$ standard error) of somatic index and transformation yields obtened in the different areas of production. Index and yields are express in percentage.

\begin{tabular}{|c|c|c|c|c|c|c|c|}
\cline { 2 - 7 } \multicolumn{1}{c|}{} & Allier & Dombes2 & Forez & Lorraine & Brenne & Poitou-C & Dombes3 \\
\hline $\begin{array}{c}\text { Coefficient } \\
\text { de condition }\end{array}$ & $\begin{array}{c}3,14 \\
\pm 0,18\end{array}$ & $\begin{array}{c}3,86 \\
\pm 0,13\end{array}$ & $\begin{array}{c}3,81 \\
\pm 0,16\end{array}$ & $\begin{array}{c}3,93 \\
\pm 0,16\end{array}$ & $\begin{array}{c}2,69 \\
\pm 0,15\end{array}$ & $\begin{array}{c}2,59 \\
\pm 0,14\end{array}$ & $\begin{array}{c}3,37 \\
\pm 0,12\end{array}$ \\
\hline $\begin{array}{c}\text { Indice viscéro- } \\
\text { somatique }\end{array}$ & $\begin{array}{c}12,59 \\
\pm 1,23\end{array}$ & $\begin{array}{c}12,45 \\
\pm 0,80\end{array}$ & $\begin{array}{c}10,78 \\
\pm 0,80\end{array}$ & $\begin{array}{c}11,44 \\
\pm 0,70\end{array}$ & $\begin{array}{c}11,50 \\
\pm 0,87\end{array}$ & $\begin{array}{c}12,38 \\
\pm 0,78\end{array}$ & $\begin{array}{c}10,36 \\
\pm 0,44\end{array}$ \\
\hline $\begin{array}{c}\text { Indice hépato- } \\
\text { somatique }\end{array}$ & $\begin{array}{c}6,02 \\
\pm 0,36\end{array}$ & $\begin{array}{c}8,71 \\
\pm 0,42\end{array}$ & $\begin{array}{c}7,66 \\
\pm 0,47\end{array}$ & $\begin{array}{c}8,78 \\
\pm 0,28\end{array}$ & $\begin{array}{c}6,04 \\
\pm 0,38\end{array}$ & $\begin{array}{c}5,29 \\
\pm 0,25\end{array}$ & $\begin{array}{c}7,38 \\
\pm 0,36\end{array}$ \\
\hline $\begin{array}{c}\text { Indice gonado- } \\
\text { somatique }\end{array}$ & $\begin{array}{c}5,77 \\
\pm 1,28\end{array}$ & $\begin{array}{c}3,28 \\
\pm 1\end{array}$ & $\begin{array}{c}2,46 \\
\pm 0,77\end{array}$ & $\begin{array}{c}2,01 \\
\pm 0,78\end{array}$ & $\begin{array}{c}4,08 \\
\pm 0,96\end{array}$ & $\begin{array}{c}5,96 \\
\pm 0,98\end{array}$ & $\begin{array}{c}2,13 \\
\pm 0,51\end{array}$ \\
\hline $\begin{array}{c}\text { Rendement au } \\
\text { filetage }\end{array}$ & $\begin{array}{c}29,67 \\
\pm 0,95\end{array}$ & $\begin{array}{c}32,09 \\
\pm 0,56\end{array}$ & $\begin{array}{c}30,23 \\
\pm 0,79\end{array}$ & $\begin{array}{c}31,73 \\
\pm 0,52\end{array}$ & $\begin{array}{c}28,44 \\
\pm 1,24\end{array}$ & $\begin{array}{c}33,19 \\
\pm 0,93\end{array}$ & $\begin{array}{c}28,67 \\
\pm 0,71\end{array}$ \\
\hline Rendement à \\
l'étêtage
\end{tabular}

\section{DISCUSSION}

Le constat d'une faible variabilité génétique des carpes issues des différentes provenances rejoint les résultats déjà obtenus par les analyses comparatives des souches d'Europe centrale (CATAUDELLA et al., 1987 ; IRNAZAROW et BIALOWAS, 1994 ; IRNAZAROW, 1995). Pour expliquer cette homogénéité génétique, l'hypothèse avancée est que les différentes provenances comparées seraient issues d'une même population d'origine entretenue à partir d'un effectif génétique faible.

Les échanges de carpes entre différentes régions de production pourraient également contribuer à la réduction des distances génétiques des souches. Une faible productivité naturelle, un élevage où la production de carpes reste secondaire ou un abandon progressif des étangs de pose privilégiant un alevinage à partir de juvéniles issus d'écloserie, sont autant de facteurs incitant l'importation de carpes et l'introgression progressive des pools géniques des souches impliquées dans ces transferts.

Les zones de production où la variabilité génétique est la plus importante (Poitou-Charentes et Dombes) sont le siège de nombreux transferts de poissons. En Dombes, les difficultés d'approvisionnement local en juvéniles ont incité pendant longtemps les structures de production à se fournir en juvéniles dans des régions traditionnellement productrices (Forez) ou disposant d'écloseries (Lorraine). De même, l'essentiel de l'alevinage des étangs de Poitou-Charentes, région récemment entrée dans une activité de production de carpes qui reste très modérée, est importé de la Brenne ou de la Lorraine. 


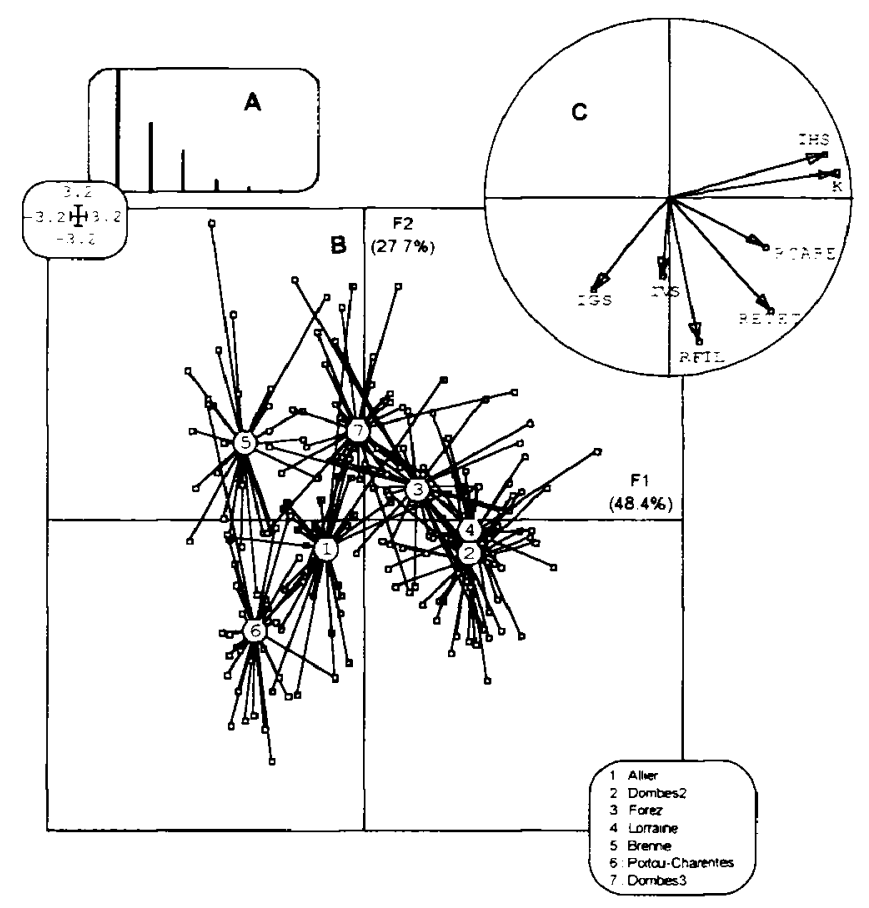

Figure 6

Résultats de l'Analyse factorielle Discriminante réalisée sur le tableau des données somatiques.

(A) Histogramme des valeurs propres.

(B) Distribution des lots dans le plan factoriel F1-F2, chaque lot est positionné au centre de gravité de ses individus.

(C) Coefficients de corrélation des variables canoniques avec les variables initiales.

Figure 6

Results of the factorial Discriminant Analysis performed on the somatic data set.

(A) Histogram of eigenvalues.

(B) Distribution of stocks on the F1-F2 factorial plane, each stock is located at the weighted average of specimens.

(C) Correlation coefficients of canonical variables with initial variables.

En revanche, les zones de production où la variabilité génétique est faible (Forez, Lorraine) correspondent davantage aux régions où les pratiques de pisciculture engendrent un brassage génétique limité. La Lorraine dispose d'une écloserie importante travaillant sur un approvisionnement en reproducteurs de carpes issus des étangs lorrains. Le Forez a été à l'origine des travaux plus ou moins empiriques de sélection sur la carpe en France, aboutissant à la " carpe royale " à partir du croisement de souches locales, tchèques et allemandes sur un critère de conformation (DE DROUIN DE BOUVILLE, 1928 a et b, 1930 ; DE NEUFBOURG, 1930 ; ROULE, 1932 ; LEVRAT, 1965). Des conditions naturelles de production particulièrement favorables ont longtemps permis à cette région d'être excédentaire pour la production de juvéniles de carpes et donc d'en fournir à la région voisine de la Dombes. Cette tendance tend à s'inverser aujourd'hui, la Dombes disposant d'une écloserie qui permet de mieux maîtriser, quantitativement, la production. 
Compte tenu des faibles distances génétiques entre les souches considérées, les différences observées pour les paramètres morphologiques, somatiques et décrivant l'aspect des filets résulteraient donc d'effets épigénétiques ou environnementaux (intensification de l'élevage, niveau des ressources alimentaires constituant les conditions d'une forte compétition interspécifique, régime thermique et caractéristiques physico-chimiques de l'eau).

Même si l'incidence des conditions d'élevage serait à étudier plus sérieusement sur le plan expérimental, le coefficient de condition et l'indice hépato-somatique, traduisant l'état nutritionnel des poissons (FAUCONNEAU et al., 1995), permettent de distinguer les lots issus des régions où l'élevage de la carpe est récent et encore peu développé (Allier, Brenne, Poitou-Charentes) des lots où l'élevage est traditionnellement centré sur cette espèce (Dombes, Forez, Lorraine).

Au niveau morphologique, seul le degré de compacité des poissons (rapport longueur sur hauteur) permet d'aboutir à une différenciation des poissons sur la base de leurs conditions d'élevage. Il apparaît en effet que les carpes ont une forme d'autant plus compacte que leurs besoins alimentaires sont satisfaits. Ces résultats seraient en accord avec des travaux déjà anciens qui illustraient le fait que des carpes sous-alimentées par suite de manque réel de nourriture, d'une trop forte densité ou d'une distribution insuffisante d'aliments de complément, ont une forme étirée (SCHÄPERCLAUS, 1962). En revanche, l'importance du développement de la tête et l'allongement dans la partie antérieure pourraient résulter d'effets génétiques ou de différences d'âge car cela n'a pas pu être relié à des conditions environnementales particulières.

Concernant les caractéristiques des filets, le degré de clarté sur la face externe et l'indice de teinte rouge sur les faces externes et internes semblent liés aux conditions environnementales. Ils permettent en effet de distinguer les lots issus de l'Allier, de la Brenne et dans une moindre mesure de Poitou-Charentes des lots issus de la Dombes ( 2 et 3 ans) et du Forez. Les filets des poissons de Poitou-Charentes et de la Lorraine se différencient par leur hauteur, en lien avec la conformation externe de ces poissons.

Dans l'ensemble, la variabilité observée sur les paramètres mesurés permet une bonne discrimination des groupes de carpes selon les conditions d'élevage. Néanmoins, aucun des critères ne permet, à lui seul, d'aboutir à une identification géographique des individus. La caractérisation des lots ne peut donc être envisagée que dans le cadre d'une approche globale reposant sur la combinaison de différents paramètres. Ainsi, en ne considérant que les variables les plus discriminantes pour chacun des lots, il est possible d'aboutir à une description type des poissons en lien avec leur provenance géographique :

- Allier (4 ans) : tête peu développée, développement en hauteur de la partie antérieure, luminance faible sur la face externe des filets, indice hépato-somatique et coefficient de condition faibles ;

- Dombes (2 ans) : tête peu développée, développement en hauteur de la partie antérieure, indice hépato-somatique et coefficient de condition élevés ;

- Dombes (3 ans) : tête bien développée, silhouette trapue malgré un faible développement en hauteur de la partie antérieure, faible rendement au filetage ;

- Forez (2 ans) : silhouette trapue, indice hépato-somatique et coefficient de condition élevés; 
- Lorraine (3 ans) : tête peu développée, silhouette trapue, développement en hauteur de la partie antérieure, hauteur des filets, indice hépato-somatique et coefficient de condition élevés ;

- Brenne (4 ans) : tête bien développée, silhouette allongée avec un faible développement en hauteur de la partie antérieure, luminance sur la face externe des filets, indice hépato-somatique, coefficient de condition et rendement au filetage faibles ;

- Poitou-Charentes (3 ans) : silhouette allongée, hauteur des filets, indice hépato-somatique et coefficient de condition faibles, rendement au filetage élevé.

Sur la base de l'ensemble des paramètres étudiés, des différences apparaissent entre les deux lots issus de la Dombes. Ce résultat pourrait être un effet de l'âge mais également une traduction des conditions d'élevage différentes pour les deux lots. En effet, les poissons de 2 ans ont été élevés en présence de silures et nourris régulièrement, alors que les poissons de 3 ans ont été élevés en polyculture classique (50-60\% de carpes, $15-20 \%$ de blancs, $10-15 \%$ de tanches et $5 \%$ de brochets) sans complément alimentaire.

\section{CONCLUSION}

Le polymorphisme enzymatique apparaît comme une bonne représentation des échanges génétiques mais ne semble pas être adapté pour caractériser différents stocks de carpes. Les techniques récentes de la génétique moléculaire fondées sur l'étude de l'ADN mitochondrial ou l'utilisation de microsatellites sur l'ADN génomique pourraient par contre fournir une meilleure description de la variabilité génétique.

Concernant les autres paramètres, des différences ont pu être notées entre les lots et permettent souvent de séparer les élevages axés sur la production de carpes (Dombes, Forez, Lorraine) et les élevages où la production de carpes restent encore secondaire (Allier, Brenne, Poitou-Charentes).

Une approche approfondie intégrant plusieurs critères pourrait sans doute permettre de préciser ces premiers résultats. Ainsi, en parallèle à ce travail, une analyse morphologique de type géométrique a permis d'aboutir à la définition d'un morphotype caractérisant les carpes de la Dombes (BOBE, 1996). De même, une relation entre la morphologie des poissons analysés après traitement automatique de l'image de leur profil (masque) et leur aptitude au filetage a pu être mise en évidence (CIBERT et al., 1999).

Cette étude illustre l'intérêt d'une approche globale dans le cadre de la comparaison de différentes populations de carpes et constitue une base de réflexion intéressante pour l'élaboration du protocole de testage de souches envisagé dans la suite du programme. Celui-ci devrait permettre de mieux évaluer l'effet des conditions d'élevage, déjà souligné par cette étude, sur les performances de croissance et l'aptitude à la transformation des carpes.

\section{REMERCIEMENTS}

Les auteurs tiennent à remercier le Ministère de l'Agriculture et de la Pêche, le Ministère de l'Enseignement Supérieur et de la Recherche et le Conseil régional de la Région Rhône-Alpes pour la participation au financement du programme « Agriculture Demain " dans lequel prend place notre étude. Nous remercions également toutes les personnes qui nous ont assisté tant sur le terrain qu'au laboratoire, particulièrement F.J. MEUNIER, C. CIBERT, B. BROHON et Y. FERMON. Enfin, nous remercions 
B. BABOUIN, Directeur de Coopepoisson, pour nous avoir mis à disposition ses installations, ainsi que Jean-Luc, pour les opérations de filetage.

\section{BIBLIOGRAPHIE}

BOBE J., 1996. Variabilité morphologique et rendements de filetage chez la carpe commune (Cyprinus carpio) : caractérisation de la population de la Dombes à taille commerciale. Mémoire ENITA Bordeaux, Prix Académie Agriculture 1996, 106 p.

BOUVET Y., SOEWARDI K., PATTEE E., 1991. The discrimination of roach Rutilus rutilus (L., 1758) populations in different parts of a river system. An investigation using biochemical markers. Hydrobiologia, 209, 161-167.

CATAUDELLA S., SOLA L., CORTI M., ARCANGELI R., LA ROSA G., MATTOCA M., COBOLLI-SBORDONI M., SBORDONI V., 1987. Cytogenetic, genic and morphometric characterization of groups of common carp, Cyprinus carpio. Selection, Hybridization and Genetic Engineering in Aquaculture. Tiews K. Schriften der Bundesforschungsan für Fischerei Publ. Berlin, Vol 1, 113-130.

CIBERT C., FERMON Y., VALLOD D., MEUNIER F.J., 1999. Morphological screening of carp Cyprinus carpio : relationship between morphology and fillet yield. Aquat. Living Resour., 12 (1), 1-10.

C.I.E., 1976. Colorimetry. Publication $n^{\circ} 15$. Bureau central du Comité International de l'Eclairage. Vienne, Autriche.

COOPER E.F., 1987. Carp in North America. Amer. Fish. Soc. Bethesta, Maryland USA, 84 p.

DE DROUIN DE BOUVILLE M.R., 1928a. Alfred MEUGNIOT (1857-1928) et la restauration de la carpiculture en France au début du XXème Siècle. Bull. Fr. Piscic., $1,2-6$.

DE DROUIN DE BOUVILLE M.R., 1928b. Alfred MEUGNIOT (1857-1928) et la restauration de la carpiculture en France au début du XXème Siècle. Bull. Fr. Piscic., 2, 30-34.

DE DROUIN DE BOUVILLE M.R., 1930. Le croisement Beauvoir n¹. Bull. Fr. Piscic., 20, 169-176.

DE NEUFBOURG, 1930. Les leçons de la campagne carpicole. Bull. Fr. Piscic., 20, 182-183.

FAUCONNEAU B., ALAMI-DURANTE H., LAROCHE M., MARCEL J., VALLOD D., 1995. Growth and meat quality relations in carp. Aquaculture, 129, 265-297.

GOULD S.J., 1966. Allometry and size in ontogeny and phylogeny. Biol. Rev., 41, 587-640.

IRNAZAROW I., 1995. Genetic variability of Polish and Hungarian carp lines. Aquaculture, $129,215-219$.

IRNAZAROW I., BIALOWAS H., 1994. Genetic characteristics of carp breding lines at the Institute of Ichthyobiology and Aquaculture of the Polish Academy of Sciences Golysz. 1 Polish lines. Acta Hydrobiol., 36, 125-142.

LEFRANC E., 1992a. Mise au point d'un outil informatique pour la saisie de la conformation de poissons vivants. Relation avec l'aptitude à la transformation. Rapport SYSAAF, Rennes, $22 \mathrm{p}$.

LEFRANC E., 1992b. ECP : Outil destiné à l'étude de la conformation de poissons vivants. Rapport DESS "Informatique appliquée aux sciences expérimentales ». Université Paul Sabatier, Toulouse, $25 \mathrm{p}$.

LE QUERE G., 1997. Situation et perspectives de la pisciculture française en étangs. Rapport Ecole Supérieure d'Agriculture d'Angers, $20 \mathrm{p}$.

LEVRAT R., 1965. La pisciculture en Dombes. Bull. Fr. Piscic., 216, 77-100. 
MEUNIER F., PASCAL M., 1981. Etude expérimentale de la croissance cyclique des rayons de nageoire de la carpe (Cyprinus carpio). Résultats préliminaires. Aquaculture, 26, 23-40.

PASTEUR G., 1985. Les paramètres statistiques communément utilisés dans l'exploitation des résultats de l'électrophorèse des protéines et leur avenir en systématique. Extrait de : M. GOYFFON, Electrophorèse et taxinomie, Société Zoologique de France, Paris.

PASTEUR N., PASTEUR G., BONHOMME F., CATALAN J., BRITTION-DAVIDIAN J., 1987. Manuel technique de génétique par électrophorèse des protéines. Technique et documentation, Lavoisier, Paris, $217 \mathrm{p}$.

PEREIRA V., 1996. Comparaison à taille commerciale, de populations de carpes communes (Cyprinus carpio) issues de différentes régions d'élevage. Variabilité morphologique et rendement au filetage. Rapport DESS “ eaux continentales : pollutions et aménagements ", Université de Besançon, $65 \mathrm{p}$.

ROULE L., 1932. Les carpes d'élevage. Bull. Fr. Piscic., 44, 229-233.

SCHÄPERCLAUS W., 1962. Traité de pisciculture en étang. In : VIGOT frères (Eds.). $603 \mathrm{p}$.

\section{Annexe 1}

Liste des variables morphologiques sélectionnées.

\section{Annex 1}

List of selected morphological variables.

\begin{tabular}{|c|l|}
\hline Abréviations & Intitulé exact de l'abréviation \\
\hline SCA & Surface carcasse \\
STE & Surface tête \\
SVI & Surface viscérale \\
SVIP & Surface viscérale postérieure \\
SVIA & Surface viscérale antérieure \\
SM & Surface musculaire \\
SMDA & Surface musculaire dorsale antérieure \\
SMDP & Surface musculaire dorsale postérieure \\
SMVP & Surface musculaire ventrale postérieure \\
SA & Surface antérieure \\
SP & Surface postérieure \\
LTE & Longueur tête \\
LA & Longueur antérieure \\
LPN & Longueur postérieure sans nageoire caudale \\
LTOT & Longueur totale \\
LST & Longueur standard \\
LTN & Longueur sans tête et sans nageoire caudale \\
LAT & Longueur antérieure sans tête \\
LP & Longueur postérieure \\
H1 & Hauteur 1 \\
H11 & Hauteur 11 \\
H12 & Hauteur 12 \\
H41 & Hauteur 4 \\
H42 & Demiteur 41 \\
H5 & Hauteur 6 \\
DH6 & Hemi hauteur 42 \\
DH42 & Hauteur 41 \\
\hline & \\
\hline
\end{tabular}




\section{Annexe 2}

Liste des variables utilisées pour décrire l'aspect des filets.

\section{Annex 2}

List of variables utilized to describe filet aspects.

\begin{tabular}{|c|l|}
\hline Abréviations & Intitulé exact de l'abréviation \\
\hline Hfil & Hauteur du filet \\
Lfil & Longueur du filet \\
Lext & Luminance face externe \\
Lint & Luminance face interne \\
$a^{*}$ ext & Coloration rouge face externe \\
$a^{*}$ int & Coloration rouge face interne \\
$b^{*}$ ext & Coloration jaune face externe \\
$b^{*}$ int & Coloration jaune face interne \\
\hline
\end{tabular}

